\title{
When discounting fails: An unexpected finding
}

\author{
DAVID ROSENFIELD \\ Southern Methodist University, Dallas, Texas 75275 \\ and \\ WALTER G. STEPHAN \\ University of Texas, Austin, Texas 78712
}

\begin{abstract}
Two studies were designed to investigate the effects of prior information about plausible causes on subsequent attributions. In the Experiment 1, prior information was given about an internal and an external cause. It was predicted and found that the stronger an expectancy for a behavior, the more the behavior would be attributed to the cause that formed the basis of the expectancy. It was also predicted that (1) for facilitative causes, the stronger the expectancy based on a given cause, the less the behavior would be attributed to other causes (discounting), and (2) for inhibitory causes, the stronger the expectancy, the more the behavior would be attributed to other causes (augmenting). These predictions were not supported. It was suggested that discounting and augmenting did not occur because subjects had been given information about both causes which "locked in" their attributions to each cause. To test this explanation, a second study was undertaken in which observers were given information about only one cause. The results of Experiment 2 indicated that discounting and augmenting may only occur for attributions to causes about which no prior information is available.
\end{abstract}

According to Kelley (1972), causal schemata are employed by attributors when two or more causal factors are perceived to interact in causing a given effect. In situations where there is more than one possible cause for an event, and when each cause alone could have caused the event, Kelley suggests that attributors employ a schema for multiple sufficient causes. In these situations, Kelley suggests that the discounting principle (Kelley, 1971) can be used to predict the attributions that will be made for the event. This principle states that the role of a given cause in producing an effect is discounted to the degree that other causes are present. Experiments conducted by Jones and others (Calder, 1974; Jones, Davis, \& Gergen, 1961; Jones, Worchel, Goethals, \& Grumet, 1971; Messick \& Reeder, 1972 ) to test the theory of correspondent inferences (Jones \& Davis, 1965) provide strong support for the discounting principle. In these experiments, subjects were given information regarding an effect (the behavior of an actor) and the state of a plausible external cause for it. They were then asked to judge the magnitude of a second possible cause. If the external cause led subjects to expect the behavior, they attributed the behavior less to a second plausible cause (dispositional characteristics of the actor) than if the external cause did not lead them to expect the behavior.

Although all of the experiments just reviewed

This research was supported by NIMH Grant 22249 to Walter G. Stephan. Requests for reprints should be sent to David Rosenfield, Department of Psychology, Southern Methodist University, Dallas, Texas 75275. The authors would like to thank Mel Snyder and Steve West for their comments on an earlier version of this manuscript. provided subjects with prior information on only one external cause, the discounting principle should also apply to situations in which the attributor has information on an internal cause or on more than one cause. In the latter situation, the degree to which a behavior is attributed to any given cause will depend upon the strength of all of the other causes.

Strength in this context refers to the expectancy that a particular behavior will occur. Attributors commonly have information on two types of causes: the dispositional characteristics of the actor and the reward contingencies of the environment. If the plausible internal cause is weak, the subsequent attributions to the external cause should be emphasized more than if the internal cause is initially strong. If a plausible external cause is weak, subsequent attributions to the internal cause will be emphasized more than if the external cause is initially strong.

The predictions emphasize an important limitation of the experiments which support the discounting principle. Typically, the subjects in these experiments are given prior information on one cause and, subsequently, their attributions to a different cause are measured. It should also be important to determine the effect of prior information concerning a particular cause on subsequent attributions to that same cause. It would be expected that there would be a positive relationship between the strength of the expectancy and subsequent attributions to the particular cause, if the behavior is consistent with the expectancy. Thus, the strength of each plausible cause has an effect on the subsequent attributions to that cause as well as on the subsequent attributions to other causes. 
To this point, the discussion has focused on plausible facilitative causes. A cause may be said to be facilitative if it would lead to a given effect. However, causes may also be inhibitory. Inhibitory causes consist of influences which would tend to decrease the probability that a particular effect would occur. When an actor behaves in a way which is inconsistent with the observer's expectancy, the observer is likely to reject as possible causes those which he believes would have inhibited the behavior. The degree to which he rejects the cause should be proportional to the strength of the cause. In addition, it seems likely that the greater the strength of an inhibitory cause, the more other causes will be emphasized. The second prediction is a rephrasing of Kelley's (1971) augmenting principle which states that the more causes existing which are inconsistent with an observed effect, the more the behavior will be attributed to other causes. The emphasis of the present prediction is on the strength of the inconsistent causes rather than on the number of causes.

To test these predictions, subjects in the present experiment were given information on an internal and an external cause prior to observing an actor behaving in a verbally aggressive manner. The actor's dispositional characteristics and the reward contingencies in the environment were described in such a way that aggressive behavior was expected (the internal and external causes were facilitative) or aggressive behavior was not expected (the internal and external causes were inhibitory). The strength of the internal and external causes was varied factorially.

\section{EXPERIMENT 1}

\section{Method}

Subjects. One hundred and twenty-five female undergraduates at the University of Texas at Austin participated in this study, in partial fulfillment of a requirement for their introductory psychology course. The data from five of the subjects were discarded because they were suspicious that the videotaped encounter group was staged. After discarding these data, the number of subjects per cell ranged from 14 to 16 .

Procedure. Subjects arrived in groups of four to seven and were greeted by one of two experimenters. The subjects were all seated in one room in front of a television screen. The experimenter told them that he was interested in how people explain the behavior of others. The experimenter then explained that he was going to show a videotape of a person ("Bob") participating in an encounter group session and that later he would ask the subjects to explain Bob's behavior. The subjects were then given information about Bob's personality and about what the group had been discussing.

The information constituted the manipulations of the internal and external causes of Bob's aggressiveness. The causes were of two types, facilitative or inhibitory, and two strengths, weak or strong. The inhibitory internal information described Bob as either (1) a member of his high school band and choir (weak expectancy) or (2) a member of his high school band and choir, and very kind, considerate, and hard to upset (strong expec-

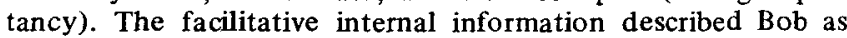
(1) a member of his high school football and boxing teams (weak expectancy) or (2) a member of his high school football and boxing teams, and a person who had tendencies toward open expressions of hostility and willingness to express his emotions in public (strong expectancy). These manipulations were based on a pretest which had revealed that the information was indeed perceived in the intended manner. In all of the internal information conditions, Bob was also described as slightly above average in intelligence and competence, and about average in creativity. Two questions were included at the end of the description of Bob. One question asked how hostile the subject thought Bob would be in a disagreement with someone else in the group. The other question asked how much the subject liked Bob. The internal information was given to the subjects before they were given the external information, in order to insure that the information about the external cause would not affect the manipulation check for the internal cause.

Each of the four external cause manipulations described the results of the group's recently finished discussion on how one should handle hostile and aggressive feelings. In all conditions, the arguments for and against expressing hostility were presented. The argument for expressing hostility was that it helps prevent the buildup of harmful inner anxiety. The argument against expressing hostility was that it could create communication barriers between people. The strength of this cause was manipulated by varying the number of people in the group who favored each side of the issue. In the inhibitory information conditions, the group's attitude was described as follows: (1) A majority of the members were against expressing hostility (weak expectancy), or (2) all of the members were against expressing hostility (strong expectancy). In the facilitative information conditions the description read: (1) A majority were in favor of expressing hostility (weak expectancy), or (2) all were in favor of expressing hostility (strong expectancy). A manipulation check was included at the end of the description of the group's attitude toward expressing hostility. It asked how rewarding the subject thought the members of the group would be toward Bob if he were to act hostilely toward another member of the group.

For half of the subjects, both the dispositional and situational information led the subjects to expect that Bob's dispositional characteristics and the attitude of the group would have facilitated his subsequent aggressive behavior, and, for the other half, both led the subjects to expect that the aggressive behavior would have been inhibited. The strength of the internal and extemal expectancies was varied factorially to produce the following four information combinations: strong intermal and strong external, strong internal and weak external, weak internal and strong external, and weak internal and weak external. Within each group, the subjects were given different combinations of the internal and external information.

After reading the information and answering the manipulation checks, the subjects viewed the videotape. At the beginning of the tape, the experimenter pointed out which person "Bob" was. After a short, friendly discussion among the members of the group, the group decided to discuss the "nature of friendship." The discussion turned into a disagreement between Bob and "Rick," in which Bob acted hostilely toward Rick. Among other things, he called Rick stupid and dumb, and said that he thought Rick had never really had a true friend and probably never would. Throughout the argument, Bob's voice volume got progressively louder until near the end, he was virtually yelling at Rick. On the other hand, although Rick never gave in to Bob's point of view, he never insulted Bob and never yelled at him.

Dependent variables. The experimenter told the subjects that he would like them to indicate how much effect each of two causes had on Bob's behavior. The causes were: (1) Bob's aggressive (or nonaggressive) nature and (2) the at titude of the group toward expressing hostility. For each of the causes, the subjects marked whether they felt the cause facilitated, inhibited, or had no effect on Bob's expression of hostility toward Rick. If the subjects felt the cause did have an effect, 
they indicated how much effect on a 23-point scale ranging from 11 (inhibited very much) to +11 (facilitated very much). After completing these questions, the subjects were debriefed.

\section{Results}

Two by two by two unweighted means analyses of variance were performed on all the data. The three independent variables were (1) type of causes (either facilitative or inhibitory), (2) strength of the information about the internal cause (weak or strong), and (3) strength of the information about the external cause (weak or strong).

Manipulation checks. The results from the manipulation checks administered after the subjects had been given information about Bob and the group, but before they saw the videotape, indicated that the manipulations were effective. The questions concerning how hostile the subjects thought Bob would be in a disagreement with someone else in the group was used as a measure of their expectancy of hostile behavior, based on Bob's dispositions. On this question, a Type of Cause by Strength of Internal Information interaction was expected, since stronger information in the facilitative condition should lead to a greater expectancy of hostile behavior, whereas stronger information in the inhibitory condition should lead to a lesser expectancy of hostile behavior. The interaction was obtained $[\mathrm{F}(1,112)=53.68, \mathrm{p}<.001$, $\mathrm{MSe}=9.23]$. The question on how rewarding the subjects thought the other members of the group would be toward Bob if he acted hostilely was used as a measure of the degree to which the external information led to an expectancy of hostile behavior from Bob. On this question, there was a significant interaction of Type of Cause by Strength of External Information $[\mathrm{F}(1,112)=3.97, \mathrm{p}<.05, \mathrm{MSe}=18.08]$. As expected, stronger information led to an increased expectancy of rewards in the facilitative condition, and to a decrease in the inhibitory condition.

Dependent variables. It was expected that, in the facilitative condition, the stronger the expectation of hostile behavior based on a given cause, the greater the attributions to that cause would be. In the inhibitory condition, it was expected that the stronger the expectation, the weaker the attributions to that cause would be. For attributions to Bob's aggressiveness (Table 1), this implies that when the causes were facilitative, strong prior information about Bob's characteristics (the

Table 1

Attributions to Bob's Aggressiveness (the Internal Cause) in Experiment 1

\begin{tabular}{ccccccc}
\hline \multirow{2}{*}{$\begin{array}{c}\text { Internal } \\
\text { Facilitative } \\
\text { Cause }\end{array}$} & \multicolumn{2}{c}{$\begin{array}{c}\text { External } \\
\text { Facilitative Cause }\end{array}$} & $\begin{array}{c}\text { Internal } \\
\text { Inhibitory }\end{array}$ & \multicolumn{2}{c}{$\begin{array}{c}\text { External } \\
\text { Inhibitory Cause }\end{array}$} \\
\cline { 3 - 3 } \cline { 6 - 7 } Cause & Strong & & Weak & Strong \\
\hline Weak & 7.1 & 7.6 & Weak & 7.4 & 7.5 \\
Strong & 9.1 & 8.6 & Strong & 5.7 & 5.7 \\
\hline
\end{tabular}

Note-The higher the number, the more the behavior was attributed to Bob's aggressiveness.
Table 2

Attributions to the Attitude of the Group (the External Cause) in Experiment 1

\begin{tabular}{|c|c|c|c|c|c|}
\hline \multirow{2}{*}{$\begin{array}{l}\text { Internal } \\
\text { Facilitative } \\
\text { Cause }\end{array}$} & \multicolumn{2}{|c|}{$\begin{array}{c}\text { External } \\
\text { Facilitative Cause }\end{array}$} & \multirow{2}{*}{$\begin{array}{l}\text { Internal } \\
\text { Inhibitory } \\
\text { Cause }\end{array}$} & \multicolumn{2}{|c|}{$\begin{array}{c}\text { External } \\
\text { Inhibitory Cause }\end{array}$} \\
\hline & Weak & Strong & & Weak & Strong \\
\hline Weak & 1.6 & 5.1 & Weak & 2.5 & -.2 \\
\hline Strong & 3.7 & 5.9 & Strong & 3.9 & .1 \\
\hline
\end{tabular}

Note-The higher the number, the more the behavior was attributed to the attitude of the group.

internal cause) should have led to greater attributions to Bob's aggressiveness than did weak prior information about Bob. When the causes were inhibitory, strong prior information about Bob's characteristics should have led to lesser attributions to Bob's aggressiveness than did weak prior information about Bob. For attributions to the attitude of the group (Table 2), it would be expected that when the causes were facilitative, strong prior information about the attitude of the group (the external cause) should have led to greater attributions to the attitude of the group than did weak prior information about the group. When the causes were inhibitory, strong prior information about the group should have led to lesser attributions to the group than did weak prior information. These predictions can be tested by the Type of Cause by Strength of Internal Information interaction on the attributions made to Bob's aggressive disposition and by the Type of Cause by Strength of External Information interaction on the attributions made to the attitude of the group. Both of these interactions were significant and had the predicted patterns $[F(1,112)=9.24, p<.005, \mathrm{MSe}=8.69$, and $\mathrm{F}(1,112)=19.39, \mathrm{p}<.001, \mathrm{MSe}=14.35]$.

It might be argued that the manipulation of dispositional information about Bob was really a manipulation of liking for Bob, rather than a manipulation of expectations of hostile behavior. Hence, the results on the attributions made to Bob's nature may have been a result of differential liking for Bob. This explanation can be tested by examining the partial correlation between the amount of hostility the subjects expected Bob to exhibit and their subsequent attributions to his aggressiveness, with the subjects' liking for Bob being factored out. This correlation was significant $(r=.26, \mathrm{p}<.005)$. In addition, the partial correlation between the subjects' liking for Bob and their attributions to his "nature" was not significant when the subject's expectation of hostile behavior was factored out $(r=-.03$, n.s. $)$. These correlations strongly suggest that the attributions made to Bob's nature were due to the amount of hostile behavior the subjects expected of Bob and not due to the degree to which they liked him.

Predictions were also made concerning how the strength of the expectancy generated by information about one cause affects the attributions made to the other cause. It was predicted that, in the facilitative 
condition, the stronger the expectancy of hostile behavior generated by information about one cause, the less the behavior should be attributed to the other cause (discounting). In the inhibitory condition, the stronger the expectancy, the more the behavior should be attributed to the other cause (similar to Kelley's augmentation principle). Thus, for attributions to Bob's aggressiveness (Table 1), when the causes were facilitative, strong information about the attitude of group (the external cause) should have led to lesser attributions to Bob's aggressiveness (the internal cause) than did weak information about the group. When the causes were inhibitory, strong information about the group should have led to greater attributions to Bob's aggressiveness than did weak information about the group. For attributions to the attitude of the group (Table 2), this implies that, when the causes were facilitative, stronger information about Bob's aggressiveness (the internal cause) should have led to lesser attributions to the attitude of the group (the external cause). When the causes were inhibitory, stronger information about Bob should have led to greater attributions to the attitude of the group. These arguments lead to the expectation of a Type of Cause by Strength of External Information interaction for attributions to Bob's aggressiveness. Similarly, a Type of Cause by Strength of Internal Information interaction is expected for attributions to the attitude of the group toward hostility. Neither of these interactions was obtained (both Fs $<1$ ).

\section{Discussion}

Support was found for the predictions about how information concerning a cause influences subsequent attributions to that cause. However, no support was found for the predictions concerning the way information about one cause affects attributions to another cause. The failure to find support for the discounting and augmenting predictions is surprising in light of the many studies cited in the introduction that have found discounting effects. One difference between the present study and the studies finding support for discounting effects stands out: In all of the previous studies, subjects were given information about only one possible cause and were then asked to make attributions to other causes. In this situation, the weaker their expectations, the more they attributed the behavior to other causes about which they had no prior information. In the present study, however, subjects were given prior information about internal and external causes. It is possible that when prior information is given about a cause, it forms an anchor point for the attributions made to that cause, and, regardless of the status of other plausible causes, the attributions to that cause are set.

Support for the suggestion that discounting and augmenting occur only for causes about which observers have no prior information comes from a study by Jones, Linder, Kiesler, Zanna, and Brehm (1968). In part of this study, Jones et al. asked observers to attribute attitudes to people who were offered varying amounts of money to perform a task for an experimenter. When the observers were not told the person's initial attitude toward the task, discounting occurred. However, when the observers were given the actor's initial attitude toward the task, discounting did not occur.

To determine whether the prior information was responsible for the failure to find discounting or aug. menting in Experiment 1, a second study was undertaken. The only difference between this study and the previous one was that in Experiment 2 subjects were given information about only one cause. Under these circumstances, discounting and augmenting should occur for attributions to the cause about which subjects are given no information.

\section{EXPERIMENT 2}

\section{Method}

Subjects. One hundred and twenty-two female undergraduates participated in the study. The subjects came from the same subject pool as those in Experiment 1. The data from three subjects were discarded: two because the subjects were suspicious that the encounter group was staged, and one because the subject did not understand the dependent measure. After discarding these data, the number of subjects per cell ranged from 13 to 17.

Procedure. The experimenter was one of the two experimenters in the previous study. The procedure was identical to that in Experiment 1, except that each subject was given only one piece of information (either internal or external), instead of two. The information given was identical to that given in Experiment 1. All of the subjects received both the external and internal manipulation checks, even though they received only one of the manipulations.

\section{Results and Discussion}

The design was a 2 by 2 by 2 factorial, with the three independent variables being (1) external or internal information, (2) facilitative or inhibitory cause, and (3) weak or strong information.

Manipulation checks. The manipulation checks showed that the manipulations were effective. For subjects given the internal information, there was a significant Type of Cause by Strength of Information interaction on the degree of hostility subjects expected Bob to exhibit $[\mathrm{F}(1,60)=23.07, \mathrm{p}<.001, \mathrm{MSe}=14.17]$. In the facilitative condition, stronger information led to greater expectations of hostile behavior, while in the inhibitory conditions, stronger information led to lesser expectations of hostile behavior. For subjects given the external information, there was a similar Type of Cause by Strength of Information interaction on the extent to which the group was expected to be rewarding toward someone expressing hostility $[F(1,51)=4.42, p<.05$, $\mathrm{MSe}=18.47]$.

Dependent variables. It was again expected that, for subjects given the internal information, there would be an interaction of Type of Cause by Strength of Cause for the subjects' attributions to Bob's nature 
Table 3

Attributions to Bob's Aggressiveness (the Internal Cause) in Experiment 2

Subjects Given Only Subjects Given Only External Information Internal Information

\begin{tabular}{lccccc}
\cline { 5 - 6 } Information & $\begin{array}{c}\text { Weak } \\
\text { Infor- } \\
\text { mation }\end{array}$ & $\begin{array}{c}\text { Strong } \\
\text { Infor- } \\
\text { mation }\end{array}$ & & $\begin{array}{c}\text { Weak } \\
\text { Infor- } \\
\text { mation }\end{array}$ & $\begin{array}{c}\text { Strong } \\
\text { Infor- } \\
\text { mation }\end{array}$ \\
\hline Facilitative & 7.6 & 7.2 & 7.5 & 8.6 \\
Inhibitory & 8.0 & 8.4 & 8.3 & 5.4 \\
\hline
\end{tabular}

Note-The higher the number, the more the behavior was attributed to Bob's aggressiveness.

(Table 3). In the facilitative condition, stronger information about Bob should have led to greater attributions to Bob's aggressiveness, while stronger information about Bob in the inhibitory condition should have led to lesser attributions to his aggressiveness. The predicted interaction was obtained $[F(1,111)=7.69, p<.01$, MSe $=7.64]$. A similar interaction was expected on the attributions to the attitude of the group by subjects given information about the group (Table 4). In the facilitative condition, stronger information about the group should have led to greater attributions to the group, while in the inhibitory condition, stronger information should have led to lesser attributions to the attitude of the group. This interaction was obtained as predicted $[F(1,111)=6.97, \mathrm{p}<.01, \mathrm{MSe}=10.30]$. These results replicate the results found in Experiment 1.

It was expected that discounting and augmenting would occur on the attributions made to the cause about which the subject had no prior information. For subjects who were given information about Bob's dispositions, the more facilitative the information, the less the behavior should have been attributed to the attitude of the group, while the more inhibitory the information, the more the behavior should have been attributed to the attitude of the group (Table 4). This leads to the expectation of a Type of Cause by Strength of Information interaction on the attributions to the attitude of the group made by subjects given dispositional information. The expected interaction was obtained $[F(1,111)=4.10, p<.05]$. A similar interaction was expected on the attributions made to Bob's aggressiveness by subjects given information about the attitude of the group (Table 3). When the causes were facilitative, stronger information about the group should have led to lesser attributions to Bob's aggressiveness, whereas when the causes were inhibitory, stronger information about the group should have led to greater attributions to Bob's aggressiveness. The differences between the means were in the predicted direction, but the interaction was not significant $(F<1)$.

The failure to find discounting and augmenting on the attributions to Bob's nature may have been due to the fact that the manipulation of the attitude of the group was not as effective as the manipulation of Bob's aggressiveness. However, it may also have been due to the subjects' inferring internal information about Bob from the external information about the group. The subjects might have felt that the more rewarding the group was toward hostile behavior, the more likely it would be that the members of the group were hostile people. This would have inhibited discounting in two ways. First, the mere fact that subjects inferred some internal information from the external information would have decreased discounting, since prior information on a cause apparently inhibits discounting on that cause. Second, if the facilitative information concerning the attitude of the group led the subjects to infer that Bob was hostile, this would have led to greater, not lesser, attributions to Bob's nature. Hence, this inference process could have nullified the tendencies toward discounting.

One way to determine whether discounting tendencies were being nullified by information on Bob's dispositions inferred from information on the attitude of the group is to control for the effect of the inferred internal information by means of a partial correlation. Rephrasing the discounting and augmenting principle in correlational terms, they state that the more the group was expected to reward hostility, the less Bob's hostile behavior should have been attributed to Bob's nature. Thus, there should be a negative partial correlation between the extent to which the subjects expected the group to be rewarding and their attributions to Bob's nature, when the effects of the amount of hostility Bob was expected to exhibit are controlled. The partial correlation was indeed negative and significant $(r=-.31$, $p<.02$ ). The correlation suggests that discounting and augmenting did occur on the attributions to Bob's nature. A parallel analysis was done for attributions to the attitude of the group. For this analysis, the partial correlation between the amount of expected hostility and subsequent attributions to the attitude of the group was computed, controlling for the extent to which the group was expected to be rewarding. The correlation also supports the discounting and augmenting hypotheses $(\mathrm{r}=-.26, \mathrm{p}<.03)$. It should be noted that the same partial correlational analysis was also performed on the data from the first study and did not demonstrate any discounting or augmenting.

Table 4

Attributions to the Attitude of the Group (the External Cause) in Experiment 2

Subjects Given Only Subjects Given Only External Information Internal Information

\begin{tabular}{lccccc} 
Information & $\begin{array}{c}\text { Weak } \\
\text { Infor- } \\
\text { mation }\end{array}$ & $\begin{array}{c}\text { Strong } \\
\text { Infor- } \\
\text { mation }\end{array}$ & & $\begin{array}{c}\text { Weak } \\
\text { Infor- } \\
\text { mation }\end{array}$ & $\begin{array}{c}\text { Strong } \\
\text { Infor- } \\
\text { mation }\end{array}$ \\
\hline Facilitative & 1.4 & 6.1 & 2.2 & .8 \\
Inhibitory & .8 & 1.1 & 1.9 & 3.9 \\
\hline
\end{tabular}

Note-The higher the number, the more the behavior was attributed to the attitude of the group. 


\section{GENERAL DISCUSSION}

The results from the present study clearly demonstrate that the strength of an attributor's prior expectancies affects the attributions which he will make. In both experiments, it was found that the strength of an expectancy based on prior information concerning one cause is an important determinant of subsequent attributions to that cause. For facilitative causes, the stronger the expectancy, the more the behavior will subsequently be attributed to that cause, while for inhibitory causes, the stronger the expectancy, the less the behavior will be attributed to that cause. These processes hold for both internal and external causes and occur regardless of whether the attributor has prior expectancies concerning one or two potential causes.

It also appears that discounting and augmenting occur only when prior information is given about one single cause. Discounting and augmenting did not occur for the attributions made to Bob's nature, or for those made to the attitude of the group, in Experiment 1 when the subjects were given prior information about an internal and an external cause. But discounting and augmenting did occur on the attributions made to Bob's nature and to the attitude of the group in Experiment 2 when the subjects had no prior information about these causes.

These results can be understood in terms of Kelley's (1972) multiple sufficient causal schema. According to this schema, when prior information is given about only one plausible cause, discounting occurs for a second cause because the more facilitative the first cause, the less facilitative the second needs to be to produce a given effect. Thus, when the cause about which prior information is given is at a highly facilitative level, a second plausible cause could be at almost any level, while when the first is at a low level, the second must be at a relatively high level. However, when prior information is given about two causes, the second cause can no longer be at almost any level. Instead, it must be at the level indicated by the prior information. Thus, changing the facilitation of the first cause does not change the perceived facilitation of the second, and discounting does not occur.

This approach may help to clarify the reason that unexpected outcomes in achievement situations are attributed to unstable causes, such as effort or luck, while expected outcomes are attributed to stable causes, such as skill and task difficulty (Frieze \& Weiner, 1971). Because unstable causal factors vary over time and across situations, one cannot have prior information about them. However, stable factors, such as skill and task difficulty, are perceived to be relatively invariant over time and across situations, and, therefore, prior information about these factors may be available. When actual performance is inconsistent with expectancies based on information about stable causes, performance will be attributed to factors about which the attributor has no prior information, that is, unstable factors. But when performance is consistent with expectancies based on the stable causes, performance will be attributed to the stable causes and the influence of the unstable causes will be discounted.

\section{REFERENCES}

CALDER, B. J. Information cues and attributions based on role behavior. Journal of Experimental Social Psychology, 1974, 10, 121-125.

Frieze, I., \& Weiner, B. Cue utilization and attributional judgments for success and failure. Journal of Personality, 1971, 39, 591-605.

Jones, E. E., \& Davis, K. E. From acts to dispositions: The attribution process in person perception. In L. Berkowitz (Ed.), Advances in experimental social psychology (Vol. II). New York: Academic Press, 1965.

Jones, E. E., Davis, K. E., \& Gergen, K. J. Role playing variations and their informational value for person perception. Journal of Abnormal and Social Psychology, $1961,63,302-310$.

Jones, E. E., Worchel, S., Goethals, G. R., \& Grumet, J. F. Prior expectancy and behavioral extremity as determinants of attitude attribution. Joumal of Experimental Social Psychology, 1971, 7, 59-80.

Jones, R. A., Linder, D. E., Kiesler, C. A., ZanNa, M., \& BREHM, J. W. Internal states or external stimuli: Observers' attitude judgments and the dissonance theory/selfpersuasion controversy. Journal of Experimental Social Psychology, 1968, 4, 247-269.

Kelley, H. H. Attribution in social interaction. Morristown, N.J: General Learning Press, 1971.

Kelley, H. H. Causal schemata and the attribution process. Morristown: N.J: General Learning Press, 1972.

Messick, D. M., \& ReEder, G. Perceived motivation, role variations, and the attribution of personal characteristics. Journal of Experimental Social Psychology, 1972, 8, $482-491$.

(Received for publication March 8, 1976; revision received June 28,1976 .) 